\title{
Diabetic Ketoacidosis Treatment Outcome and Associated Factors Among Adult Patients Admitted to the Emergency Department and Medical Wards at King Abdulaziz Medical City, Riyadh, Saudi Arabia
}

Abdulaziz Alotaibi ${ }^{1}$, Abdulrahman Aldoukhi ${ }^{1}$, Bayan Albdah ${ }^{2}$, Jamila A. Alonazi ${ }^{3}$, Amjad S. Alseraya ${ }^{3}$, Najla Alrasheed ${ }^{3}$

1. Medicine, King Saud Bin Abdulaziz University for Health Sciences, Riyadh, SAU 2. Biostatistics and Bioinformatics, King Abdullah International Medical Research Center, Riyadh, SAU 3. Internal Medicine, King Abdulaziz Medical City, Riyadh, SAU

Corresponding author: Najla Alrasheed, rasheedn@ngha.med.sa

\section{Abstract}

\section{Background}

Diabetic ketoacidosis (DKA) is a life-threatening condition with high morbidity and mortality rates. It should be diagnosed immediately and managed intensively to prevent its significant complications.

\section{Objectives}

The aim of this study to assess DKA treatment outcome and associated factors among adult patients at King Abdulaziz Medical City Emergency Department and Medical Wards, Riyadh, Saudi Arabia.

\section{Materials and Methods}

A retrospective cross-sectional study was conducted using a chart review to assess DKA treatment outcome and associated factors. All patients who were admitted as DKA cases from September 2017 to August 2019 were selected by simple random sampling except those with incomplete charts or younger than 14 years. Data were entered and analyzed using SAS Version 9.4 (SAS Institute, Cary, NC, USA).

\section{Results}

A total of 223 reviewed charts were collected. The frequency of DKA recurrence in most of the patients was once per year (126 [56.5\%]). The most common precipitating factor was inappropriate insulin therapy (104 [46.64\%]). More than half of the patients (120 [53.81\%]) got out of DKA management protocol within 24-72

Received 08/19/2020 Review began 08/19/2020 Review ended 08/20/2020 Published 08/27/2020

\section{() Copyright 2020}

Alotaibi et al. This is an open access article distributed under the terms of the Creative Commons Attribution License CC-BY 4.0., which permits unrestricted use, distribution, and reproduction in any medium, provided the original author and source are credited. hours with a hospital stay of less than or equal to five days. The mortality rate was $1.83 \%$. Patients with two or more DKA episodes per year tended to be admitted to ICU more frequently than those with one episode $(\mathrm{p}=0.001)$. It was found that patients who had a duration of one to five years of diabetes mellitus were almost five times more likely to get out of DKA in more than 72 hours when compared with those who had a duration of more than five years (adjusted OR: 4.7 ; $95 \%$ CI: $1.34-16.60$; $\mathrm{p}=0.01$ ).

\section{Conclusions}

The findings of this study highlight that majority of DKA patients showed improvement and discharged with a very low mortality rate. Inappropriate insulin therapy was the most common precipitating factor; thus, educating diabetic patients about the complications of treatment non-compliance is an important part of management.

Categories: Endocrinology/Diabetes/Metabolism, Internal Medicine, Medical Education

Keywords: diabetic ketoacidosis (dka), hospital stay, hospital mortality, treatment outcome, icu admission

\section{Introduction}

Diabetic ketoacidosis (DKA) is one of the life-threatening acute hyperglycemic complications of diabetes mellitus (DM) carrying high morbidity and mortality among type 1 diabetics and less commonly but recognized in type 2 diabetics. It is characterized by massive hyperglycemia, ketonemia, and acidosis [1]. It is most commonly precipitated by infections, non-compliance to insulin therapy, or first presentation of diabetes. Other factors include stressors such as cerebrovascular accidents, cardiac ischemia, trauma, and pancreatitis [1-3]. 
DKA can result in significant complications as volume depletion and severe acidosis may lead to cardiac arrest and acute kidney injury (AKI); thus, prompt diagnosis and initiation of intensive treatment protocol by experienced staff are important for successful management $[3,4]$ followed by careful monitoring to prevent the occurrence of iatrogenic complications with insulin and fluid administration, such as hypoglycemia, hypokalemia, and cerebral edema. Appropriate early fluid therapy and insulin administration show a significantly better outcome and fewer recurrences. DKA can be successfully managed within 12-36 hours with appropriate treatment, with which the complications and mortality can be prevented and reduced effectively [5].

To address this, a few studies were conducted to draw the actual picture regarding the DKA outcome and their risk factors in Saudi Arabia [6,7]. Therefore, this study helps health care workers to build a uniform treatment protocol. The gained information will help also both patients and health care providers to know the most common reasons for DKA recurrence to prevent future episodes. Furthermore, the results of this study can be used as a database for future researches. Keeping the aforesaid facts in view, this study aims to assess DKA treatment outcome and associated factors among adult patients at King Abdulaziz Medical City (KAMC) Emergency Department and Medical Wards, Riyadh, Saudi Arabia.

\section{Materials And Methods}

This retrospective cross-sectional study used a chart review to assess DKA treatment outcome and associated factors among adult patients at KAMC Emergency Department and Medical Wards. KAMC is a tertiary hospital located in Riyadh, the capital city of Saudi Arabia. It has a bed capacity of 690 beds and provides all types of care to all National Guard soldiers and their families, starting from primary health care up to tertiary specialized care.

To determine the sample size, the following assumptions were considered in this study. According to a study was conducted in Ethiopia to assess the DKA outcome, $84.90 \%$ of the patients were discharged with improvement with 95\% confidence level and 5\% margin of error [8]. The sample size was calculated by using Raosoft software (http://www.raosoft.com/samplesize.html). Based on this, the final expected sample size was approximately 196 patients. All patients who were admitted as DKA cases from September 1, 2017, to August 31, 2019, were included in the study, whereas patients with incomplete chart or younger than 14 years were excluded.

Simple random sampling was used to collect the data for both independent and outcome variables from BESTcare by using a checklist that was adapted from a related previous study [8]. Data were collected by the co-authors and data collectors. The collected data were checked for accuracy and completeness daily by the principal investigator. To identify any potential problems, the checklist was tested before the actual data collection on $10 \%$ of the total sample size, and some modifications were considered accordingly. The checklist included socio-demographic characteristics, complications, comorbidities, precipitating factors, frequency, clinical presentation, biochemical tests, renal function, electrolyte, complete blood count, and management protocol of DKA patients. Then, the collected data were used for analysis to assess DKA treatment outcome and associated factors.

Data were presented as mean \pm standard deviation. Frequencies and percentages were used to describe categorical variables. We used Fisher's exact test or chi-square test for association between categorical variables, and the Wilcoxon two-sample test, Kruskal-Wallis test (for not normally distributed data), t-test, or one-way analysis of variance (ANOVA) test (for normally distributed data) for continuous variables. We examined outcome "time within which patients get out of DKA" predictors using multivariate logistic regression. The regression model included age, type of DM, duration of DM, treatment regimen for DM, and comorbidities such as hypertension, dyslipidemia, and chronic kidney disease (CKD). All statistical tests were considered significant at $\mathrm{p}<0.05$. Data were analyzed using the statistical program SAS Version 9.4 (SAS Institute, Cary, NC, USA).

Ethical approval was obtained from the Institutional Review Board at King Abdullah International Medical Research Center.

\section{Results}

\section{Demographics and clinical characteristics}

A total of 223 reviewed charts were collected from the BESTcare system, of which 106 (47.53\%) were males and 117 (52.47\%) were females, and almost $60.54 \%$ of them were in the age group of $14-30$ years. Out of the reviewed patients, 166 (74.44\%) had type 1 diabetes and 57 (25.56\%) had type 2 diabetes. Majority (184 [82.51\%]) of the patients were on insulin treatment, and the other patients were on either oral (13 [5.83\%]), both oral and insulin $(19$ [8.52\%]), or not on any medication (7 [3.14\%]). More than half of the patients had DM for more than five years (125 [56.05\%]). Although most of the patients had no other chronic comorbidities, hypertension and dyslipidemia were documented in $52(23.32 \%)$ and $50(22.42 \%)$ patients, respectively. Table 1 shows the demographics and clinical characteristics profile of all respondents. 


\section{Cureus}

Variable

Age of the patient

14-30 years

$30.1-40$ years

40.1-50 years

50.1-60 years

$>60$ years

Sex of the patient

Male

Female

Educational background

Elementary school

Tertiary school

Bachelor's degree

Master's degree

Unknown

Marital status

Single

Married

Widowed

Divorce

Unknown

Type of DM

Type 1

Type 2

Duration of DM

1-5 years

$>5$ years

Newly diagnosed

Not documented

Treatment regimen for DM

Insulin

Oral

Both

Not on any medications

Comorbidity

Hypertension

No

Yes
N

(\%)

60.54

11.21

7.17

8.52

12.56

47.53

52.47

4.93

7.17

1.35

0.45

86.10

52.02

32.74

1.79

0.90

12.56

74.44

25.56

19.73

56.05

6.28

17.94

82.51

5.83

8.52

3.14

7

171

76.68

52

23.32 


\section{Cureus}

\section{Dyslipidemia}

No

Heart failure

No

TABLE 1: Demographics and clinical characteristics $(n=223)$

DM, diabetes mellitus; ACS, acute coronary syndrome; CKD, chronic kidney disease; BA, bronchial asthma

\section{The frequency of recurrent DKA and precipitating factors}

The frequency of DKA recurrence per year was assessed and showed that most of the patients had DKA once (126 [56.5\%]), 61 (27.35\%) had two episodes, and 36 (16.14\%) had three or more episodes. Inappropriate insulin therapy was the most common precipitating factor in 104 (46.64\%) of the patients, which was mainly due to poor insulin compliance. Infections such as urinary tract infection, gastroenteritis, and upper and lower respiratory tract infections were the second most common precipitating factors in 70 (31.39\%) of the DKA cases. Other precipitating factors are shown in Table 2. 


\section{Cureus}

Variable

Frequency of recurrent DKA per year

Once

Twice

$\geq 3$

Precipitating factors

Inappropriate insulin therapy

Infection (urinary tract infection, respiratory tract infection, acute gastroenteritis, skin infection, etc.)

AMI

Sepsis of any origin

Pancreatitis

CVA

Drugs (steroids, thiazides, pentamidine, second-generation anti-psychotics, cocaine, immune checkpoints inhibitors, SGLT2 inhibitors)

Emotional stress

Any recent surgical intervention ( $<1$ month)

Acute renal failure

Inappropriate diet

First presentation of DM

Unknown/not documented

Reasons for discontinued insulin

Lack of supplies

Fade up of daily injection/poor compliance/missed doses

Combination of reasons

Insulin pump not working

Not documented

Type of infection

UTI

URTI

\section{TABLE 2: The frequency of recurrent DKA and precipitating factors $(n=223)$}

DKA, diabetic ketoacidosis; AMI, acute coronary syndrome; CVA, cerebrovascular accident; SGLT2, sodium-glucose co-transporter-2; UTI, urinary tract infection; URTI, upper respiratory tract infection; LRTI, lower respiratory tract infection

\section{Clinical presentation of patients who were admitted with DKA}

DKA patients were most commonly presented with abdominal pain (143 [64.13\%]) and vomiting (139 [62.33\%]). In addition, nausea (89 [39.91\%]), polyuria/polydipsia (42 [18.83\%]), and malaise (31 [13.90\%]) 


\section{Cureus}

were reported fairly by DKA patients. On physical examination, 141 (63.23\%) of admitted patients with DKA were found to be looking ill with dehydration signs such as dry mucous membrane, poor skin turgor, sunken eyes, tachycardia, and hypotension. Regarding vital signs, the mean body temperature was $36.8 \pm 0.48^{\circ} \mathrm{C}$, pulse rate was $106.14 \pm 19.49$ beats $/ \mathrm{min}$, and systolic and diastolic blood pressures were $119.99 \pm 17.15 \mathrm{mmHg}$ and $67.88 \pm 12.56 \mathrm{mmHg}$, respectively. Table 3 shows the details regarding the clinical presentation of patients.

\section{Symptoms of DKA}

Variable

Polyuria/polydipsia

No

Yes

Nausea

No

Yes

Vomiting

Weight loss

Malaise

Abdominal pain

Yes

\section{Anorexia}

No

SOB

LOC

Weakness

Signs of DKA

Looking ill 


\section{Cureus}

Dehydration signs (dry mucous. poor skin turgor, sunken eyes, tachycardia, hypotension)

Constitutional signs

Kussmaul respiration

Ketone breath

No

Altered mental status

No

\section{TABLE 3: : Symptoms and signs of patients who admitted with DKA ( $n=223)$}

\section{Laboratory values of admitted patients with DKA}

The average levels of random blood glucose, serum potassium, serum bicarbonate, and white blood cell (WBC) count were $27.65 \pm 9.8 \mathrm{mmol} / \mathrm{L}, 4.94 \pm 0.85 \mathrm{mmol} / \mathrm{L}, 13 \pm 6.56 \mathrm{mEq} / \mathrm{L}$, and $13.27 \pm 6.43\left(\mathrm{x} 10^{9} / \mathrm{L}\right)$, respectively. Besides, the average calculated anion gap for the chart-reviewed patients was $26.79 \pm 6.45$, and the average urine ketones level was $109 \pm 51.3 \mathrm{mg} / \mathrm{dL}$. Table 4 shows the details of the laboratory results. 


\section{Cureus}

\section{Variable}

Random blood glucose level (mmol/L)

Serum creatinine $(\mu \mathrm{mol} / \mathrm{L})$

Serum BUN (mmol/L)

Serum sodium $(\mathrm{mmol} / \mathrm{L})$

Serum potassium (mmol/L)

Anion gap calculation $(\mathrm{mmol} / \mathrm{L})$

Serum bicarbonate $(\mathrm{mEq} / \mathrm{L})$

Urinary ketones $(\mathrm{mg} / \mathrm{dL})$

Serum amylase (U/L)

WBC count $\left(x 10^{9} / \mathrm{L}\right)$

RBC count $\left(x 10^{12} / \mathrm{L}\right)$

Hemoglobin (g/L)

Platelets $\left(x 10^{9} / \mathrm{L}\right)$

\section{Mean \pm SD}

$27.65 \pm 9.8$

$130.13 \pm 102.43$

$7.3 \pm 5.83$

$132.42 \pm 5.43$

$4.94 \pm 0.85$

$26.79 \pm 6.45$

$13 \pm 6.56$

$109.95 \pm 51.3$

$60.61 \pm 100.58$

$13.27 \pm 6.43$

$4.97 \pm 0.78$

$137.95 \pm 25.03$

$340.67 \pm 120.16$

TABLE 4: Laboratory values of admitted patients with DKA $(n=223)$

BUN, blood urea nitrogen; WBC, white blood cell; RBC, red blood cell; DKA, diabetic ketoacidosis

\section{Management protocol and outcome of DKA patients}

While the most commonly used type of fluid bolus was $0.9 \%$ normal saline (218 [97.76\%]) with an average amount of $1 \pm 0.08$ liters, fluid maintenance was mostly with 5\% dextrose in water (DW5\%) (173 [77.58\%]). The rate of fluid maintenance in majority (160 [71.75\%]) of the patients was $100-120 \mathrm{~mL} /$ hour. Regular insulin was the only type of insulin used in all DKA patients with an average administration rate of 5.56 \pm 2.23 U/hour in the first 24 hours. Most (208 [93.69\%]) of the patients were repleted with intravenous potassium chloride. The majority of DKA patients got out of DKA within 24-72 hours (120 [53.81\%]) and less than 24 hours (76 [34.08\%]). Nearly three-quarters of patients 170 (76.23\%) did not develop any complications with DKA; however, AKI and hypokalemia were the most common complications in 30 (13.45\%) and 8 (3.59\%) of the patients, respectively. Most (152 [68.16\%]) of the patients were treated in the general medical wards without the need for intensive care unit (ICU) admission, whereas the others (71 [31.84\%]) were admitted to ICU mainly due to either severity of the illness, comorbidities, and/or developed complications. Almost 97 (43.50\%) and 85 (38.12\%) of the patients had a hospital stay of one to two days and three to five days, respectively. Concerning the treatment outcome of DKA patients, only four (1.79) patients showed no improvement and died, whereas the other patients (219 [98.21\%]) showed improvement and discharged. Table 5 shows the details of the management protocol and outcome of DKA patients.

\begin{tabular}{|c|c|c|}
\hline Variable & $\mathbf{N}$ & (\%) \\
\hline \multicolumn{3}{|l|}{ Type of IV fluid bolus } \\
\hline Did not receive bolus because of overload & 2 & 0.90 \\
\hline DW5\% & 3 & 1.35 \\
\hline NS & 218 & 97.76 \\
\hline Amount of IV fluid bolus (in liters) (mean \pm SD) & $1 \pm 0.08$ & \\
\hline \multicolumn{3}{|l|}{ Type of fluid maintenance used in the management } \\
\hline DW5\% & 173 & 77.58 \\
\hline NS & 48 & 21.52 \\
\hline RL & 2 & 0.90 \\
\hline
\end{tabular}




\section{Cureus}

Rate of fluid maintenance used in the management

\begin{tabular}{|c|c|c|}
\hline $60-70 \mathrm{~mL} / \mathrm{h}$ & 8 & 3.59 \\
\hline $70-100 \mathrm{~mL} / \mathrm{h}$ & 33 & 14.80 \\
\hline $100-120 \mathrm{~mL} / \mathrm{h}$ & 160 & 71.75 \\
\hline$>120 \mathrm{~mL} / \mathrm{h}$ & 21 & 9.42 \\
\hline Patient refused and signed against medical advice & 1 & 0.45 \\
\hline Insulin administration rate in the first 24 hours $(\mathrm{U} / \mathrm{h})$ & $5.56 \pm 2.23$ & \\
\hline Regular & 223 & 100.0 \\
\hline \multicolumn{3}{|l|}{ Time within which patients get out of DKA } \\
\hline $24 \mathrm{~h}$ & 76 & $34 . C$ \\
\hline$>24 \mathrm{~h}$ and $<72 \mathrm{~h}$ & 120 & 53.81 \\
\hline$>72 \mathrm{~h}$ & 27 & \\
\hline
\end{tabular}

Potassium replacement

No

Yes

Complications of DKA

No complication

Hypoglycemia

Hypokalemia

Non-anion gap hyperchloremic acidosis

Cerebral edema/brain injury

Acute respiratory distress syndrome

Acute renal failure

Cardiac arrest

Others

Admitted to ICU

No

Hospital stay

1-2 days

3-5 days

$>5$ days

$41 \quad 18.39$

General treatment outcome of DKA patients

Shows improvement and discharged

TABLE 5: Management protocol of DKA patients $(n=223)$ 


\section{Cureus}

\section{Comparison among admitted ICU patients and associated factors}

Patients with a frequency of recurrent DKA two times or more tended to be admitted to ICU more frequently than those who had only one episode per year $(\mathrm{p}=0.001)$. In addition, patients who were admitted with Kussmaul sign had a propensity to be admitted to ICU than those who were not $(\mathrm{p}=0.005)$. In addition, those who showed altered mental status at the time of presentation were likely to be admitted to ICU ( $p=0.002)$. Regarding the vital signs, patients with an initial pulse rate of $113.42 \pm 18.68$ beats $/$ min tended to be admitted to the ICU when compared with those with an initial pulse rate of $102.65 \pm 18.95$ beats $/ \mathrm{min}(p \leqslant 0.0001)$. Regarding the initial lab results, patients who presented with an investigation lab result of anion gap calculation of $28.24 \pm 7.01 \mathrm{mmol} / \mathrm{L}$ ( $\mathrm{p}-0.03)$, serum bicarbonate of $9.61 \pm 7.49 \mathrm{mEq} / \mathrm{L}(\mathrm{p} \leqslant 0.0001)$, and WBC count 1 of $6.72 \pm 6.02 \times 10^{9} / \mathrm{L}(\mathrm{p} \leqslant 0.0001)$ were more likely to be admitted to ICU than the other group (26.11 $\pm .08 \mathrm{mmol} / \mathrm{L}, 14.6 \pm 5.4 \mathrm{mEq} / \mathrm{L}$, and $11.67 \pm 5.98 \times 10^{9} / \mathrm{L}$, respectively). The time within which the patients got out of DKA was found to be longer ( $>24$ hours) in ICU patients when compared with those who got a DKA free in less than 24 hours ( $\leqslant \leqslant 0.0001)$. Also, those patients who were admitted to the ICU appeared to have a prolonged hospital stay (>five days) when compared with those who only stayed for less than two days $(p \leqslant 0.0001)$. Table 6 shows the details for the comparison of response among patients who were admitted to ICU.

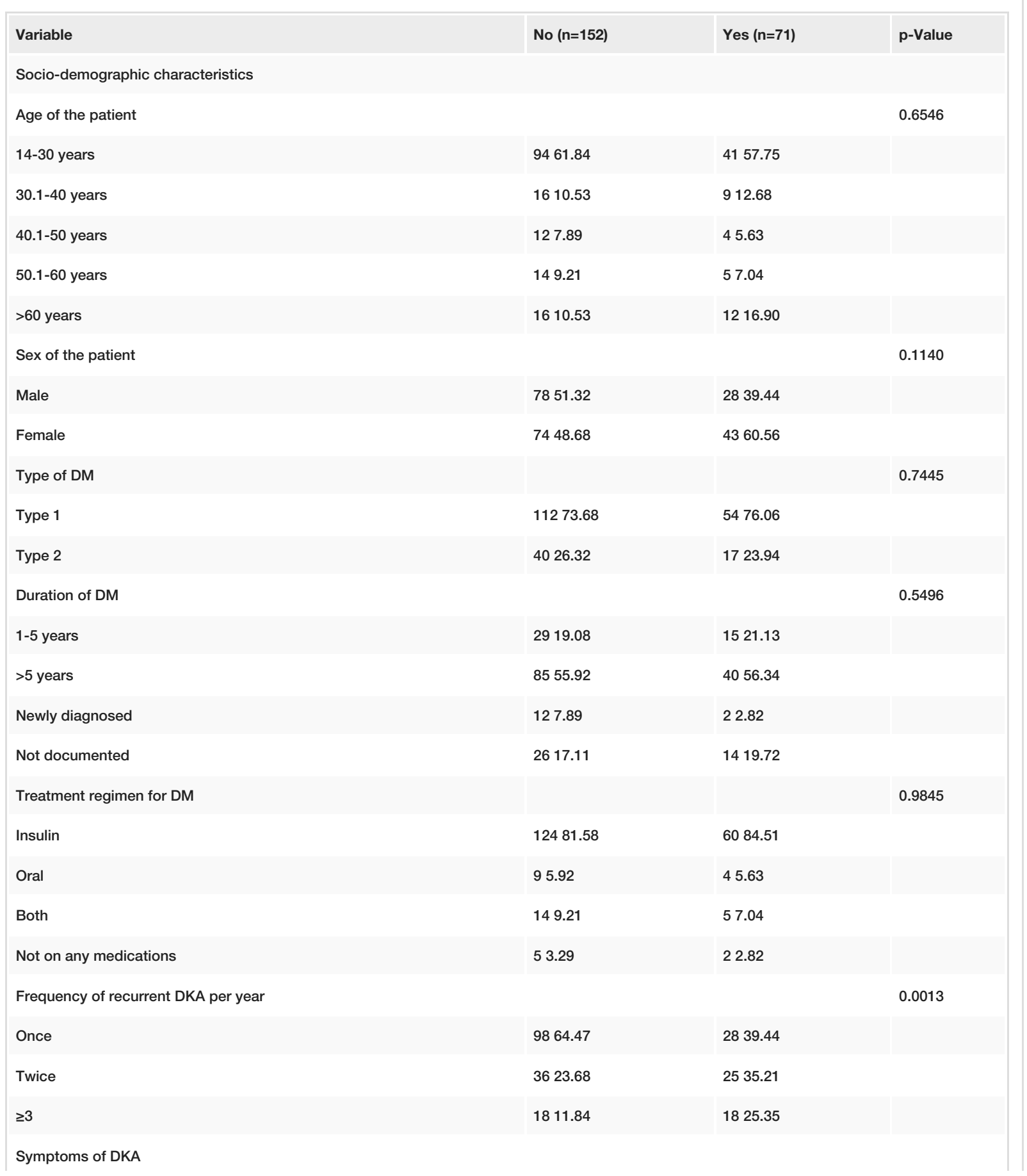




\section{Cureus}

Polyuria/polydipsia

0.1411

No

11978.29

6287.32

Yes

3321.71

912.68

Nausea

0.7696

No

9059.21

4461.97

Yes

6240.79

2738.03

Vomiting

0.4601

No

6039.47

2433.80

Yes

9260.53

4766.20

Weight loss

0.6822

No

Yes

14897.37

6895.77

42.63

34.23

Abdominal pain

0.7646

No

5636.84

9663.16

2433.80

Yes

SOB

4766.20

No

Yes

13689.47

5780.28

1610.53

1419.72

LOC

No

14494.74

6287.32

Yes

85.26

912.68

Signs of DKA

Dehydration signs

0.0751

No

6240.79

2028.17

Yes

9059.21

5171.83

Constitutional signs

No

Yes

12984.87

2315.13

Kussmaul respiration

No

Yes

14998.03

31.97

Ketone breath

15199.34

No

Yes

10.66

Altered mental status

No

14394.08

Yes

Temperature

95.92

$36.93 \pm 0.47$

$120.36 \pm 17.77$

Systolic blood pressure $(\mathrm{mmHg})$

6692.96

57.04

0.0053

6388.73

811.27

0.2385

6997.18

22.82

0.0018

0.1276

5678.87

1521.13

$36.85 \pm 0.48$

0.5509

$119.18 \pm 15.84$

0.7131 


\section{Cureus}

\begin{tabular}{|c|c|c|c|}
\hline Diastolic blood pressure $(\mathrm{mmHg})$ & $67.8 \pm 12.06$ & $68.07 \pm 13.64$ & 0.8681 \\
\hline Pulse rate in (beats $/ \mathrm{min}$ ) & $102.65 \pm 18.95$ & $113.42 \pm 18.68$ & \\
\hline \multicolumn{4}{|l|}{ Laboratory values of admitted patients with DKA } \\
\hline Random blood glucose level (mmol/L) & $26.88 \pm 9.17$ & $29.31 \pm 10.9$ & 0.1649 \\
\hline Serum creatinine $(\mu \mathrm{mol} / \mathrm{L})$ & $129.12 \pm 111.89$ & $132.31 \pm 79.13$ & 0.0623 \\
\hline serum BUN (mmol/L) & $7.2 \pm 5.55$ & $7.53 \pm 6.41$ & 0.8655 \\
\hline Serum sodium (mmol/L) & $131.88 \pm 5.4$ & $133.59 \pm 5.35$ & 0.0086 \\
\hline Serum potassium (mmol/L) & $4.84 \pm 0.8$ & $5.15 \pm 0.93$ & 0.0069 \\
\hline Anion gap calculation (mmol/L) & $26.11 \pm 6.08$ & $28.24 \pm 7.01$ & 0.0287 \\
\hline Serum bicarbonate (mEq/L) & $14.6 \pm 5.4$ & $9.61 \pm 7.49$ & \\
\hline Urinary ketones (mg/dl) & $108.02 \pm 50.81$ & $114.16 \pm 52.49$ & 0.2713 \\
\hline Serum amylase (U/L) & $63.91 \pm 119.99$ & $54.32 \pm 45.47$ & 0.5402 \\
\hline WBC count $\left(\times 10^{9} / \mathrm{L}\right)$ & $11.67 \pm 5.98$ & $16.72 \pm 6.02$ & \\
\hline RBC count (x10 $12 / L)$ & $5.01 \pm 0.73$ & $4.89 \pm 0.87$ & 0.4863 \\
\hline Hemoglobin (g/L) & $139.74 \pm 22.32$ & $134.11 \pm 29.83$ & 0.3528 \\
\hline Platelets (x109/L) & $326.98 \pm 109.46$ & $370.4 \pm 136.81$ & 0.0141 \\
\hline \multicolumn{4}{|l|}{ Management outcome of DKA patients } \\
\hline \multicolumn{4}{|l|}{ Time within which patients get out of DKA } \\
\hline$=24 \mathrm{~h}$ & 7046.05 & 68.45 & \\
\hline$>24 \mathrm{~h}$ and $<72 \mathrm{~h}$ & 7046.05 & 5070.42 & \\
\hline$>72 \mathrm{~h}$ & 127.89 & 1521.13 & \\
\hline \multicolumn{4}{|l|}{ Hospital stay } \\
\hline $1-2$ days & 9159.87 & 68.45 & \\
\hline $3-5$ days & 4630.26 & 3954.93 & \\
\hline$>5$ days & 159.87 & 2636.62 & \\
\hline General treatment outcome of DKA patients & & & 0.0963 \\
\hline Shows improvement and discharged & 15199.34 & 6895.77 & \\
\hline Shows no improvement (referred or died) & 10.66 & 34.23 & \\
\hline
\end{tabular}

TABLE 6: Comparison among admitted ICU patients and associated factors $(n=223)$

DM, diabetes mellitus; DKA, diabetic ketoacidosis; BUN, blood urea nitrogen; WBC, white blood cell; RBC, red blood cell

\section{Predictors of time within which the patient gets out of DKA}

Different factors have been studied as determinants of time within which the patient gets out of DKA. It was found that patients who had a duration of one to five years of DM were almost five times more likely to get out of DKA in more than 72 hours when compared with those who had a duration of more than five years (adjusted OR: 4.7 ; 95\% CI: 1.34-16.60; $\mathrm{p}=0.01$ ). Patients who had a treatment regimen of both oral medication and insulin were likely four times to get free of DKA in more than 72 hours as compared with those only were on insulin (adjusted OR: 4.5 ; 95\% CI: 0.97-21.15; $p=0.05$ ). Patients with CKD had a likelihood to get out of DKA in more than 72 hours by more than four times as compared with those who were not (adjusted OR: $4.0 ; 95 \%$ CI: 0.86-18.68; $\mathrm{p}=0.04$ ). Table 7 shows the predictors of time within which the patients got out of DKA. 


\section{Cureus}

\begin{tabular}{|c|c|c|c|c|c|c|c|c|c|c|}
\hline \multirow{2}{*}{ Independent variable } & \multirow{2}{*}{$\begin{array}{l}<72 \mathrm{~h} \\
(\mathrm{n}=196)\end{array}$} & \multirow{2}{*}{$\begin{array}{l}>72 \mathrm{~h} \\
(\mathrm{n}=27)\end{array}$} & \multirow{2}{*}{$\begin{array}{l}\text { OR } \\
\text { "univariate" }\end{array}$} & \multicolumn{2}{|l|}{$95 \% \mathrm{Cl}$} & \multirow{2}{*}{$\begin{array}{l}\text { p- } \\
\text { Value }\end{array}$} & \multirow{2}{*}{$\begin{array}{l}\text { Adjusted OR } \\
\text { "multivariate" }\end{array}$} & \multicolumn{2}{|l|}{$95 \% \mathrm{Cl}$} & \multirow{2}{*}{$\begin{array}{l}\mathrm{p}- \\
\text { Value }\end{array}$} \\
\hline & & & & Lower & Upper & & & Lower & Upper & \\
\hline Age (ref: $14-30$ years) & 12463.27 & 1140.74 & & & & & & & & \\
\hline 30.1-40 years & 2211.22 & 311.11 & 1.537 & 0.397 & 5.958 & 0.5339 & 1.137 & 0.216 & 5.987 & 0.8792 \\
\hline 40.1-50 years & 136.63 & 311.11 & 2.601 & 0.642 & 10.535 & 0.1803 & 1.548 & 0.253 & 9.474 & 0.6365 \\
\hline $50.1-60$ years & 178.67 & 27.41 & 1.326 & 0.271 & 6.501 & 0.7278 & 0.496 & 0.060 & 4.066 & 0.5136 \\
\hline$>60$ years & 2010.20 & 829.63 & 4.510 & 1.617 & 12.581 & 0.0040 & 0.816 & 0.113 & 5.914 & 0.8404 \\
\hline Type of DM (ref: type 1) & 15177.04 & 1555.56 & & & & & & & & \\
\hline Type 2 vs type 1 & 4522.96 & 1244.44 & 2.684 & 1.172 & 6.149 & 0.0195 & 0.693 & 0.149 & 3.210 & 0.6387 \\
\hline $\begin{array}{l}\text { Duration of DM (ref: >5 } \\
\text { years) }\end{array}$ & 11659.18 & 933.33 & & & & & & & & \\
\hline $1-5$ years & 3718.88 & 725.93 & 2.438 & 0.849 & 7.001 & 0.0977 & 4.720 & 1.342 & 16.600 & 0.0156 \\
\hline Newly diagnosed & 136.63 & 13.70 & 0.991 & 0.116 & 8.461 & 0.9937 & 1.708 & 0.113 & 25.758 & 0.6989 \\
\hline Not documented & 3015.31 & 1037.04 & 4.297 & 1.603 & 11.517 & 0.0038 & 3.691 & 1.138 & 11.969 & 0.0296 \\
\hline $\begin{array}{l}\text { Treatment regimen for DM } \\
\text { (ref: insulin) }\end{array}$ & 16684.69 & 1866.67 & & & & & & & & \\
\hline Both (oral and insulin) & 136.63 & 622.22 & 4.257 & 1.442 & 12.570 & 0.0087 & 4.523 & 0.967 & 21.155 & 0.0552 \\
\hline Not on any medications & 63.06 & 13.70 & 1.537 & 0.175 & 13.491 & 0.6981 & 2.240 & 0.136 & 36.934 & 0.5728 \\
\hline Oral & 115.61 & 27.41 & 1.677 & 0.344 & 8.167 & 0.5223 & 1.161 & 0.175 & 7.689 & 0.8769 \\
\hline Hypertension (ref: no) & 15880.61 & 1348.15 & & & & & & & & \\
\hline Yes vs no & 3819.39 & 1451.85 & 4.478 & 1.945 & 10.310 & 0.0004 & 3.205 & 0.849 & 12.105 & 0.0858 \\
\hline Dyslipidemia (ref: no) & 15880.61 & 1555.56 & & & & & & & & \\
\hline Yes vs no & 3819.39 & 1244.44 & 3.327 & 1.440 & 7.687 & 0.0049 & 2.050 & 0.628 & 6.691 & 0.2342 \\
\hline CKD (ref: no) & 18996.43 & 2281.48 & & & & & & & & \\
\hline Yes vs no & 73.57 & 518.52 & 6.136 & 1.794 & 20.986 & 0.0038 & 4.005 & 0.861 & 18.632 & 0.0369 \\
\hline
\end{tabular}

TABLE 7: Predictors of time within which the patient got out of DKA $(n=223)$

DM, diabetes mellitus; CKD, chronic kidney disease; DKA, diabetic ketoacidosis

\section{Discussion}

Majority (98.21\%) of the admitted DKA patients showed improvement and discharged. This result is much higher than a study conducted at Adama University Hospital, where only $84.90 \%$ showed improvement and were discharged [8]. In this study, the mortality rate is $1.83 \%$, which is relatively close to that reported in a study conducted at Chang Gung Memorial Hospital, where only $0.67 \%$ of the admitted patients with DKA showed no improvement and died [9]. The mortality rate in this study is considered relatively low as compared to other studies that were conducted in Zambia and Malaysia with a mortality rate of $16.66 \%$ and $17.6 \%$, respectively $[10,11]$. Concerning the hospital stay, majority of the patients had a hospital stay of less than or equal to five days, which is considered less than a study that was conducted locally at King Fahad Medical City with an average hospital stay of seven days [12]. This difference in improvement, mortality, and length of hospital stay can be explained mainly by the advanced age, associated comorbidities of the patients, and the different treatment protocols that were provided by different medical institutions.

In this study, the frequency of DKA recurrence in majority of patients was found to be only one episode per year. This shows lower recurrence as compared with a study was conducted at Adama University Hospital, in which the recurrence was found to be two or more episodes per year in most (65\%) of the patients [8]. It is well known worldwide that the most common precipitating factor for DKA is infection followed by inappropriate insulin therapy $[2,8]$. In contrast, the most common precipitating factor in our study is 
inappropriate insulin therapy (46.64\%) due to poor compliance followed by infections (31.39\%). Also, this was reported in other various studies that were conducted in Saudi Arabia, which showed a similar pattern in poor insulin compliance, which was the most common among patients with recurrent DKA admissions [12-15]. This variation in precipitating factors was attributed to the difference in population across the world as explained in an article review that was published by Kitabchi et al. [1]. Further researches are needed to find out the reason behind fewer recurrence of DKA that is precipitated by inappropriate insulin therapy as found in our study.

Concerning the clinical features of DKA patients, abdominal pain (69\%) was reported among the patients in a study published in 2015 at KAMC [15]. Vomiting (61.6\%) was reported in another local study that was conducted among DKA adults in a tertiary hospital in Riyadh, Saudi Arabia [14]. Polyurea (26.3\%) and polydipsia (28.2\%) were found as initial clinical presentations among patients. In addition, dehydration signs including hypotension and tachycardia were reported in $61.8 \%$ of the patients in a study conducted locally at King Fahad Medical City [12]. These clinical features are consistent with our results.

Nearly three-quarters of the patients who were admitted with DKA did not develop complications associated with the disease. Majority (13.45\%) of our patients were complicated with AKI, which is considered low as compared to a study conducted in Bangladesh showing that $29.5 \%$ of their patients developed AKI [16]. This variation could be explained by the severity of dehydration and how early fluid replacement is initiated. We found that $3.59 \%$ of our patients were complicated with hypokalemia, which is consistent with various studies reporting the incidence of hypokalemia in DKA patients to range from $0 \%$ to $4 \%$ during various stages of DKA management $[17,18]$. This is explained by insulin administration and correction of acidosis and hyperosmolarity that drive potassium intracellularly, resulting in hypokalemia; thus, careful monitoring of potassium is an important aspect of management [19].

Most of the DKA patients in our study were treated in the general medical wards (68.16\%), which is consistent with a study conducted at Auckland Hospital in which almost 70\% did not need to be admitted to ICU [20]. Similarly, other studies showed that more than half of their DKA patients were treated in the general medical wards [21,22]. Similar to a Libyan study, we found that patients who were admitted to ICU either due to severity of the episode, development of acute complications, or having other comorbidities stayed significantly longer in hospital (>five days) when compared to those who were treated in the general medical wards (<two days) [21].

With appropriate treatment of DKA, patients are expected to be out of DKA, which is defined as glucose less than $200 \mathrm{mg} / \mathrm{dL}$ and at least two of the following: venous $\mathrm{pH}$ over 7.30, serum bicarbonate more than or equal to $15 \mathrm{mEq} / \mathrm{L}$, or anion gap less than or equal to $12 \mathrm{mEq} / \mathrm{L}$ within 48 hours [23,5]. In KAMC, the treatment protocol is generally according to the recent American Diabetic Association guidelines for DKA management [24]. The majority of DKA patients in our study got out of DKA within 24-72 hours (53.81\%) or less than 24 hours (34.08\%). We found that patients who were already diagnosed with DM and being treated with both oral and insulin were more likely to be out of DKA in more than 72 hours when compared to those who were on insulin alone. This can be explained by the concept that patients who are on both oral and insulin therapy are likely to be type 2 diabetics with advanced or uncontrolled disease and thus higher rates of complications and comorbidities. We could not find studies that support this result; thus, further studies are needed to identify factors that play a role in a prolonged time to be out of DKA.

Each study has its limitations. A limitation of our study is that we did not have much data regarding the treatment protocol in detail due to a poor chart review in this part. Therefore, we could not assess the management and its associated factors. Another limitation is that our study was only limited to one hospital (KAMC) and not generalized to other hospitals in the Riyadh region.

\section{Conclusions}

The findings of this study highlight that majority of DKA patients showed improvement and were discharged with a very low mortality rate. Most of the patients received their medical treatment in medical wards, with a hospital stay of less than or equal to five days. Our data suggest that the time within which the patient got out of DKA is 24-72 hours. The frequency of DKA recurrence was found to be only one episode per year and precipitated commonly by poor insulin therapy and infections. Further studies are needed to better understand the management and its associated factors.

\section{Additional Information \\ Disclosures}

Human subjects: Consent was obtained by all participants in this study. King Abdullah International Medical Research Center issued approval RC19/390/R. Ethical approval was obtained from the Institutional Review Board at King Abdullah International Medical Research Center (Study Number RC19/390/R). Animal subjects: All authors have confirmed that this study did not involve animal subjects or tissue. Conflicts of interest: In compliance with the ICMJE uniform disclosure form, all authors declare the following: Payment/services info: All authors have declared that no financial support was received from any 
organization for the submitted work. Financial relationships: All authors have declared that they have no financial relationships at present or within the previous three years with any organizations that might have an interest in the submitted work. Other relationships: All authors have declared that there are no other relationships or activities that could appear to have influenced the submitted work.

\section{Acknowledgements}

We would like to thank the following medical students for taking part in the data collection process and contributed substantially to the work: Sawsan Alharthi, Lamiaa Alanzan, Raghad Alshaalan, Majd Binkhunain, and Nermeen Alrajhi.

\section{References}

1. Kitabchi AE, Umpierrez GE, Murphy MB, Barrett EJ, Kreisberg RA, Malone JI, Wall BM: Management of hyperglycemic crises in patients with diabetes. Diabetes Care. 2001, 24:131-53. 10.2337/diacare.24.1.131

2. Kitabaci AE, Umpierrez GE, Murphy MB, Kreisberg RA: Hyperglycaemic crises in adult patients with diabetes: a consensus statement from the American Diabetes Association. Diabetes Care. 2006, 29:2739-48. 10.2337/dc06-9916

3. Hifumi T, Kiriu N, Kato H, Inoue J, Koido Y: Survival after prolonged resuscitation from cardiac arrest due to diabetic ketoacidosis using extracorporeal life support. Am J Emerg Med. 2013, 31:8921-2. 10.1016/j.ajem.2012.12.041

4. Hursh BE, Ronsley R, Islam N, Mammen C, Panagiotopoulos C: Acute kidney Injury in children with type 1 diabetes hospitalized for diabetic ketoacidosis. JAMA Pediatr. 2017, 171:1700-20. 10.1001/jamapediatrics.2017.0020

5. Kitabchi AE, Umpierrez GE, Murphy MB, et al.: Hyperglycemic crises in diabetes . Diabetes Care. 2004, 27:94102. 10.2337/diacare.27.2007.s94

6. Alahmadi S, Ragaban A, Alblowi S, Aljumail E, Zarif H: Diabetic ketoacidosis; annual incidence and precipitating factors at King Abdulaziz Medical City, Jeddah. Egypt J Hosp Med. 2018, 72:4831-6.

7. Al-Hayek AA, Robert AA, Braham RB, Turki AS, Al-Sabaan FS: Frequency and associated risk factors of recurrent diabetic ketoacidosis among Saudi adolescents with type 1 diabetes mellitus. Saudi Med J. 2015, 36:216-20. 10.15537/smj.2015.2.10560

8. Kassaye D, Girsha W, Guto G, Deybasso H: Diabetic ketoacidosis treatment outcome and associated factors among adult patients admitted to medical wards of Adama Hospital Medical College, Oromia, Ethiopia. Am J Intern Med. 2018, 6:34-42. 10.11648/j.ajim.20180602.12

9. Lin SF, Lin JD, Huang YY: Diabetic ketoacidosis: comparisons of patient characteristics, clinical presentations and outcomes today and 20 years ago. Chang Gung Med J. 2005, 28:24-30.

10. Usman A, Syed Sulaiman SA, Khan AH, Adnan AS: Profiles of diabetic ketoacidosis in multiethnic diabetic population of Malaysia. Trop J Pharm Res. 2015, 14:179-85. 10.4314/TJPR.V14I1.25

11. Mahesh MG, Rajendra PS, Chandra BJS, Sajed S: The study of the different clinical patterns of diabetic ketoacidosis and common precipitating events and independent mortality factors. J Clin Diagn Res. 2017, 11:42-6. 10.7860/JCDR/2017/25347.9760

12. Almalki MH, Buhary BM, Khan SA, Almaghamsi A, Alshahrani F: Clinical and biochemical characteristics of diabetes ketoacidosis in a tertiary hospital in Riyadh. Clin Med Insights Endocrinol Diabetes. 2016, 9:7-11. 10.4137/CMED.S39639

13. Qari FA: Precipitating factors for diabetic ketoacidosis . Saudi Med J. 2002, 23:173-6.

14. Al-Rubeaan KA, Aftab SA, Alotaibi MS, Alghamdi AA, Rafiullah MR: Clinico-laboratory characteristics of diabetic keto acidosis in adults in a tertiary hospital in Saudi Arabia. Eur Rev Med Pharmacol Sci. 2011, 15:1202-6.

15. Naeem MA, Al-Alem HA, Al-Dubayee MS, et al.: Characteristics of pediatric diabetic ketoacidosis patients in. Saudi Arabia. Saudi Med J. 2015, 36:20-5. 10.15537/smj.2015.1.9763

16. Rahim MA, Rouf R, Ahmed AU, Mitra P, Zaman S, Uddin KN, Latif Z: Clinical characteristics and outcome of diabetic ketoacidosis: experience at BIRDEM, Dhaka, Bangladesh. Crit Care J. 2015, 3:53-6. 10.3329/bccj.v3i2.25110

17. Adrogué HJ, Lederer ED, Suki WN, Eknoyan G: Determinants of plasma potassium levels in diabetic ketoacidosis. Medicine (Baltimore). 1986, 65:163-72. 10.1097/00005792-198605000-00004

18. Manzano F, Kozak GP: Acute quadriplegia in diabetic hyperosmotic coma with hypokalemia . JAMA. 1969, 207:2278-81. 10.1001/jama.1969.03150250108018

19. Gosmanov A.R. Gosmanova E.O. Dillard-Cannon E: Management of adult diabetic ketoacidosis. Diabetes Metab Syndr Obes. 2014, 7:255-64. 10.2147/DMSO.S50516

20. Bagg W, Sathu A, Streat S, Braatvedt GD: Diabetic ketoacidosis in adults at Auckland Hospital, 1988-1996. Aust N Z J Med. 1998, 28:604-8. 10.1111/j.1445-5994.1998.tb00655.x

21. Elmehdawi RR, Ehmida M, Elmagrehi H, Alaysh A: Incidence and mortality of diabetic ketoacidosis in Benghazi-Libya in 2007. Oman Med. 2013, 28:178-83. 10.5001/omj.2013.50

22. Guisado-Vasco P, Cano-Megías M, Carrasco-de la Fuente M, Corres-González J, Matei AM, GonzálezAlbarrán O: Clinical features, mortality, hospital admission, and length of stay of a cohort of adult patients with diabetic ketoacidosis attending the emergency room of a tertiary hospital in Spain. Endocrinol Nutr. 2015, 62:277-84. 10.1016/j.endonu.2015.02.003

23. Kitabchi AE, Umpierrez GE, Miles JM, Fisher JN: Hyperglycemic crises in adult patients with diabetes . Diabetes Care. 2009, 32:1335-43. 10.2337/dc09-9032

24. American Diabetes Association: 14. Diabetes care in the hospital: Standards of Medical Care in Diabetes2018. Diabetes Care. 2018, 41:144-51. 10.2337/dc18-S014 